\title{
Neutron-antineutron oscillation search with MicroBooNE and DUNE
}

\author{
Yeon-jae Jwa ${ }^{a, *}$ and for the MicroBooNE and DUNE Collaborations \\ ${ }^{a}$ Columbia University, \\ New York, NY, 10027, USA \\ E-mail: yj2429@columbia.edu
}

The Deep Underground Neutrino Experiment (DUNE) is an international project planned for measurements in neutrino physics and astrophysics and searches for phenomena predicted by theories Beyond the Standard Model (BSM). The excellent imaging capability of the Liquid Argon Time Projection Chamber (LArTPC) technology DUNE employs, in particular particle tracking and identification in the DUNE Far Detector (FD), as well as the FD size and underground placement, allow the experiment to achieve high sensitivity to various rare processes. More specifically, BSM theories predict the existence of baryon number non-conservation effects, in particular processes where the baryon number (B) changes by two units. Here, we discuss the sensitivity of DUNE to neutron-antineutron oscillation $(\Delta B=2)$. With full event simulation and reconstruction using the LArSoft package, we have investigated the background to potential signal events from atmospheric neutrino interactions and particle misidentification, and utilized machine learning techniques to enhance the discrimination between signal and background. The methodologies being developed for a high-sensitivity search for neutron-antineutron oscillation in DUNE can also be demonstrated with the currently running, albeit much smaller, MicroBooNE LArTPC. We discuss progress on demonstrating the developed techniques with the first-ever search for neutron-antineutron oscillation in a LArTPC using a small sample of MicroBooNE data.

40th International Conference on High Energy physics - ICHEP2020

July 28 - August 6, 2020

Prague, Czech Republic (virtual meeting)

\footnotetext{
${ }^{*}$ Speaker
} 


\section{Introduction}

Neutron-antineutron oscillation is a process where a neutron transforms itself to its antiparticle (antineutron), and it is predicted by theories beyond the Standard Model [1,2]. This process does not conserve baryon number, changing baryon number by two units $(\Delta B=2)$. According to Sakharov's well-known argument [3], baryon number violation (BNV) is one of the necessary conditions to account for the matter-dominated universe we observe, and neutron-antineutron oscillation process could explain this baryon dominance [4]. Because the neutron is charge-neutral, neutron-antineutron oscillation, while violating baryon number, does not change electric charge (a fundamental quantum number). Neutral particle-antiparticle oscillations can also be observed within Standard Model physics in neutral meson states; $K^{0}-\bar{K}^{0}, B^{0}-\bar{B}^{0}$ oscillations.

There are two experimental methods to search for neutron-antineutron oscillation: using free neutrons and using bound neutrons. In the free neutron method, a neutron beam is propagated to a distant target, looking for interactions between the target material and antineutrons. The current best $90 \%$ C.L. limit for the neutron-antineutron oscillation lifetime using the free neutron method comes from the ILL/Grenoble experiment, and corresponds to $8.6 \times 10^{7} \mathrm{~s}$ [5]. A future experiment using this method is planned at the European Spallation Source (ESS), aiming for improved sensitivity [6]. In the bound neutron method, one instead searches for an antineutron's annihilation signature in a nucleus, and in a neutron-abundant environment (i.e. in large particle detectors). The neutronantineutron oscillation lifetime in a nucleus is related to the free neutron-antineutron oscillation lifetime through an intranuclear suppression factor $R$, with the following equation:

$$
\tau_{n-\bar{n}}^{2}=\frac{T_{n-\bar{n}}}{R}
$$

where $\tau_{n-\bar{n}}[\mathrm{~s}]$ is the free neutron-antineutron oscillation lifetime, $T_{n-\bar{n}}[\mathrm{~s}]$ is the bound neutronantineutron oscillation lifetime, $R\left[\mathrm{~s}^{-1}\right]$ is the intranuclear suppression factor. The intranuclear suppression factor was recently calculated for ${ }^{40} \mathrm{Ar}$ to be $R=5.6 \times 10^{22} \mathrm{~s}^{-1}$ [7], although the work presented in Sec. 3 uses $R=6.66 \times 10^{22} \mathrm{~s}^{-1}$, the intranuclear suppression factor for ${ }^{56} \mathrm{Fe}$. The current best $90 \%$ C.L. limit of neutron-antineutron oscillation lifetime using the bound neutron method comes from Super-K, and corresponds to $3.6 \times 10^{32}$ years for oxygen-bound neutrons. This is equivalent to $4.7 \times 10^{8} \mathrm{~s}$ for the free neutron-antineutron oscillation lifetime [8].

The signature of neutron-antineutron oscillation for bound neutrons can be understood as an intranuclear annihiliation of an antineutron and a nearby nucleon; proton or neutron. The momentum sum of final state particles is nearly 0, limited by initial nucleon Fermi motion and final state intra-nuclear interactions, and the total energy release amounts to approximately twice the nucleon mass. Most of the final states with significant branching ratios for these annihilation processes $(\bar{n}, n),(\bar{n}, p)$, contain multiple charged and neutral pions, with the spherical symmetry for outgoing particles. This leads to a characteristic 'star-like' topology of neutron-antineutron oscillation. Liquid Argon Time Projection Chambers (LArTPCs), equipped with millimeter scale position resolution and good calorimetric resolution, are well-suited instruments for capturing this distinctive signal topology of neutron-antineutron oscillation, by stereoscopically imaging the interaction. 


\section{Image Analysis Based Search with LArTPCs}

The neutron-antineutron oscillation search analysis presented here was carried out for DUNE and MicroBooNE, which share the same LArTPC technology. While DUNE and MicroBooNE face different backgrounds and operation principles, the analysis methodology using convolutional neural network (CNN)-based image classification in combination with conventional reconstructed kinematic variables for Boosted Decision Tree (BDT) classification was commonly used.

\subsection{Neutron-antineutron oscillation searches in DUNE and MicroBooNE}

In DUNE, 10 years of exposure with a total of $40 \mathrm{kt} \mathrm{TPC} \mathrm{fiducial} \mathrm{volume} \mathrm{in} \mathrm{all} \mathrm{four} \mathrm{of} \mathrm{the}$ FD modules are used for sensitivity studies of rare searches. Over the 10 years of operation, self-triggering on rare and off-beam events such as proton-decay, neutron-antineutron oscillation, and atmospheric neutrinos with $>99 \%$ trigger efficiency is assumed. As the FD modules are a mile underground, they are mostly shielded from cosmogenic activity, and fiducialization further can reject cosmic muons close to the active volume surfaces; this makes atmospheric neutrino interactions the only likely background for this search. The Bartol flux at the Soudan Mine location [9] was used in the calculation of the event rate of atmospheric neutrino interactions in DUNE. Including atmospheric neutrino oscillations, the rate of atmospheric neutrino interactions was estimated at 288.6 events per kton·year.

In MicroBooNE, cosmogenic activity is constant and abundant because the detector operates on the earth's surface. Thus, the major background for MicroBooNE's search is cosmogenic interactions, which also coincide with the signal activity in the TPC readout, due to the detector's long TPC exposure. The MicroBooNE detector is $80 \mathrm{t}$ in TPC volume, which is about 500 times smaller than DUNE FD modules' combined fiducial volume. The total combined exposure of MicroBooNE off-beam data collected using a random trigger over its entire operation is on the order of hours.

\subsection{CNN and BDT combined classification}

The strategy of searching for neutron-antineutron oscillation in DUNE and MicroBooNE includes a convolutional neural network (CNN)-based image classification and a BDT-based classification. In both DUNE and MicroBooNE, the TPC readout windows were treated as 2D images in wire-channel axis and time-tick axis, and used for CNN classification. The CNNs for DUNE and MicroBooNE, respectively, were trained to distinguish images containing signal neutron-antineutron oscillation from images containing atmospheric (for DUNE), and cosmogenic background (for MicroBooNE). The output $\mathrm{CNN}$ score in combination with reconstructed kinematic quantities were used as the input variables of the BDT classification in a (BDT+CNN) "combined analysis" $[10,11]$. This combined analysis aims to make the most in selecting the neutron-antineutron oscillation signal from the background, by making the CNN learn the topological features of the signal and background images, and by making the BDT learn the differences in kinematics between the signal and background interactions. Alternatively, a traditional BDT-only analysis or CNN-only analysis is possible. For the event reconstruction, Projection Matching algorithms (PMA) [12] was used for DUNE, and Pandora pattern recognition [13] was used for MicroBooNE. 


\section{Sensitivity Projections for a Future Search with DUNE}

With the combined analysis, a signal efficiency of $8 \%$ and a background efficiency of $0.02 \%$ were achieved [10], when an optimized BDT cut was applied on the BDT response shown in Fig. 1. A $0.02 \%$ efficiency to the background event rate, corresponds to 20 background events for 400 kton-year of exposure.

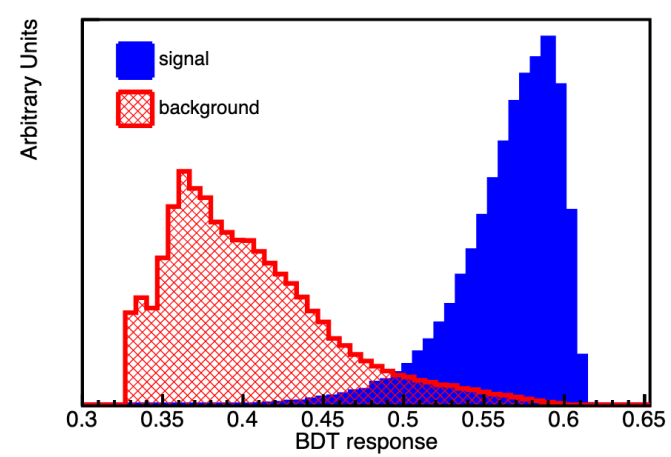

Figure 1: BDT response distributions for neutron-antineutron oscillation signal (shown as blue histogram) and background atmospheric neutrino interactions (shown as red histogram). (The figure is excerpted from [10].)

A preliminary sensitivity to neutron-antineutron oscillation lifetime in DUNE was calculated with the given exposure ( $E$ in (neutron-years)), signal efficiency $(\epsilon)$, and expected background count $(b)$, along with corresponding uncertainties. Equation 2 represents the probability distribution of the intranuclear total decay width $(\Gamma)$ of the neutron-antineutron oscillation process for a given observed number of events $(n)$. Here, a Bayesian assumption was followed, where $P(E), P(\epsilon)$, and $P(b)$ are Gaussian priors with the uncertainties of $E$, $\epsilon$, and $b, P(\Gamma)$ is a flat distribution for $\Gamma \geq 0$ and 0 for negative $\Gamma$.

$$
P(\Gamma \mid n)=A \iiint \frac{e^{-(\Gamma \epsilon E+b)}(\Gamma \epsilon E+b)^{n}}{n !} P(\Gamma) P(E) P(\epsilon) P(b) d E d \epsilon d b .
$$

The value of $\Gamma_{0.9}$ that covers $90 \%$ of the total integral domain in the integral Eq. 2 can be found using:

$$
90 \%=\frac{\int_{\Gamma=0}^{\Gamma_{0.9}} P(\Gamma \mid n) d \Gamma}{\int_{\Gamma=0}^{\infty} P(\Gamma \mid n) d \Gamma} .
$$

Then, the $90 \%$ C.L. value for the $n-\bar{n}$ oscillation lifetime is a reciprocal of $\Gamma_{0.9}$ :

$$
T_{n-\bar{n}}=\frac{1}{\Gamma_{0.9}} .
$$

With this calculation, the $90 \%$ C.L. sensitivity for the argon-bound neutron-antineutron oscillation lifetime was estimated at $6.45 \times 10^{32}$ years for an exposure of $1.33 \times 10^{35}$ neutron-years in DUNE, under the uncertainty assumptions of $3 \%, 25 \%, 25 \%$ for exposure, signal efficiency, and background rate, respecticely. When translated into the free neutron-antineutron oscillation lifetime, 
this sensitivity corresponds to $5.53 \times 10^{8} \mathrm{~s}$. The result is comparable with the current best limit at Super-K, which is $3.6 \times 10^{32}$ years for bound, or $4.7 \times 10^{8} \mathrm{~s}$ for free neutron-antineutron oscillation lifetime[8]. Effort on further improving the analysis and sensitivity continues. The systematic uncertainties due to the choice of generators and intranuclear modeling are being evaluated, while the uncertainty assumptions for the exposure, the signal efficiency, and the background rate will be re-evaluated after a thorough study.

\section{Ongoing Proof-of-concept Search with MicroBooNE}

While MicroBooNE is not sensitive to compete with the current existing neutron-antineutron oscillation lifetime limits, it can serve as a test-bed for neutron-antineutron oscillation searches in LArTPC's, including the future search in DUNE. Analysis strategies (combined, BDT-only, CNN-only) in Sec. 2 were demonstrated on MicroBooNE simulations, and tested on a small subset of MicroBooNE data [11]. Figure 2 shows the curves for the background rejection rate and the signal efficiency for these analysis methods on simulated samples. The combined analysis showed improved sensitivity over BDT-only analysis, and comparable sensitivity to the $\mathrm{CNN}$-only analysis.

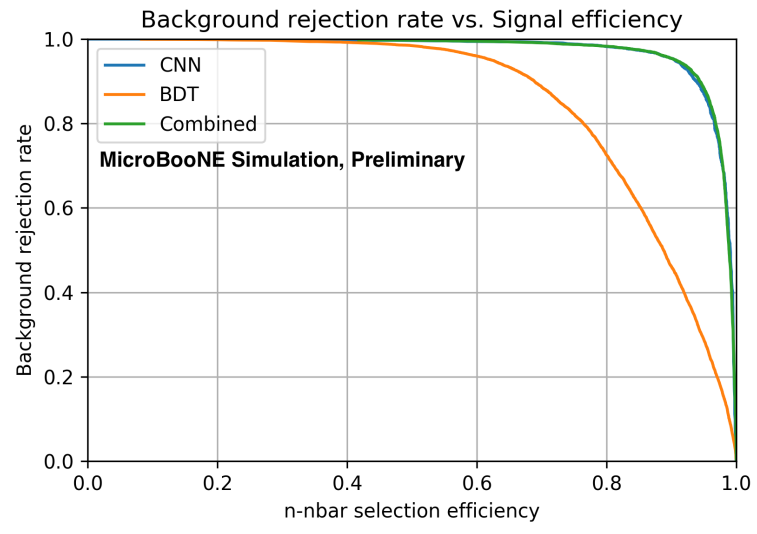

Figure 2: Background rejection rate versus neutron-antineutron oscillation signal efficiency achieved with the BDT-only(orange), CNN-only (blue), and BDT+CNN combined (green) analyses. Signal efficiencies are $62.3 \%, 90.5 \%, 90.7 \%$ for BDT-only, CNN-only, and BDT+CNN combined methods at $95 \%$ background rejection. (Figure is excerpted from [11].)

When applied to a small subset of MicroBooNE off-beam data, a data-to-Monte Carlo simulation discrepancy in BDT response and CNN response were observed [11]. This is understood to be due to imperfect modeling of cosmic flux and detector response. Improved data-to-Monte Carlo agreement is being targeted with ongoing analysis development.

\section{Summary}

Future large-scale, deep underground liquid argon time projection chambers, such as those proposed for DUNE, provide an opportunity for background-free searches for neutron antineutron oscillations, due to their excellent interaction imaging capabilities. With use of deep learning tools, 
the future DUNE is well positioned to provide world-leading sensitivity for neutron-antineutron oscillation. MicroBooNE, sharing the same technology as DUNE, is well-positioned to provide a proof-of-principle demonstration of such analysis methods, and the first LArTPC-based search for neutron-antineutron oscillation.

\section{References}

[1] R. N. Mohapatra and R. E. Marshak, Local BL Symmetry of Electroweak Interactions, Majorana Neutrinos, and Neutron Oscillations, Phys. Rev Lett., 44, 1316, 1980.

[2] D. G. Phillips et al., Neutron-Antineutron Oscillations:Theoretical Status and Experimental Prospects, Phys. Repts., 612, 1-45, 2016.

[3] A. D. Sakharov, Violation of CP Invariance, $C$ asymmetry, and baryon asymmetry of the universe, Sov. Phys. Usp., 34(5), 392-393, 1991.

[4] V. Kuzmin, CP violation and baryon asymmetry of the universe, JETP Lett., 12, 228 (1970).

[5] M. Baldo-Ceolin, A new experimental limit on neutron-antineutron oscillations, Z. Phys. C, 63, 409 (1994).

[6] A. Addazi et al., New high-sensitivity searches for neutrons converting into antineutrons and/or sterile neutrons at the European Spallation Source, arXiv:2006.04907.

[7] J. L. Barrow, E. S. Golubeva, E. Paryev, J.-M. Richard, Progress and simulations for intranuclear neutron-antineutron transformations in ${ }_{18}^{40} \mathrm{Ar}$, Phys. Rev. D, 101(3), 036008, 2020.

[8] Super-Kamiokande Collaboration, K. Abe et al., Neutron-Antineutron Oscillation Search at Super-Kamiokande. June 22 to July 2, 2020, Neutrino 2020.

https://nusoft. fnal.gov/nova/nu2020postersession/pdf/posterPDF-43.pdf.

[9] G. Barr, T. K. Gaisser, T. Stanev, Flux of atmospheric neutrinos, Phys. Rev. D, 39, 3532, 1989.

[10] DUNE Collaboration, B. Abi et al., Deep Underground Neutrino Experiment (DUNE), Far Detector Technical Design Report, Volume II DUNE Physics, arXiv:2002.03005.

[11] MicroBooNE Collaboration, R. Acciarri et al., Progress Toward the First Search for Bound Neutron Oscillation into Antineutron in a Liquid Argon TPC, MICROBOONE-NOTE-1093-PUB

[12] M. Antonello et al., Precise 3D track reconstruction algorithm for the ICARUS T600 liquid argon time projection chamber detector, Adv. High Energy Phys. 2013(2013) 260820, [arXiv:1210.5089].

[13] MicroBooNE Collaboration, R. Acciarri et al., The Pandora multi-algorithm approach to automated pattern recognition of cosmic-ray muon and neutrino events in the MicroBooNE detector, Eur. Phys. J. C, 78(1), 82, 2018. 